Revue européenne des sciences sociales

European Journal of Social Sciences

52-1 | 2014

Politiques du libre accès en sciences humaines et sociales

\title{
Cognition et formation académique
}

Les professeurs de science de la vie et de la terre face au « problème des éléphants »

\section{Gérald Bronner}

\section{(2) OpenEdition}

\section{Journals}

Édition électronique

URL : http://journals.openedition.org/ress/2698

DOI : $10.4000 /$ ress. 2698

ISSN : 1663-4446

Éditeur

Librairie Droz

Édition imprimée

Date de publication : 6 mai 2014

Pagination : 139-161

ISBN : 978-2-600-01829-6

ISSN : 0048-8046

Référence électronique

Gérald Bronner, "Cognition et formation académique », Revue européenne des sciences sociales [En ligne], 52-1 | 2014, mis en ligne le 12 mai 2014, consulté le 19 avril 2019. URL : http:// journals.openedition.org/ress/2698; DOI : 10.4000/ress.2698 


\title{
COGNITION ET FORMATION ACADÉMIQUE LES PROFESSEURS DE SCIENCE DE LA VIE ET DE LA TERRE FACE AU «PROBLĖME DES ÉLÉPHANTS》
}

GÉRALD BRONNER

Université Paris-Diderot - LIED gerald.bronner@univ-paris-diderot.fr

\begin{abstract}
Résumé. Certaines théories scientifiques ont du mal à s'imposer au sens commun, c'est notamment le cas pour la théorie de l'évolution de Darwin. Cette résistance varie en fonction des bassins culturels concernés (elle s'exprime différemment en France et aux États-Unis, par exemple), mais partout où elle se produit, elle est adossée à des raisonnements captieux plus ou moins implicites. Cet article est basé sur une expérience in vitro realisée avec 60 enseignants de science de la vie et de la terre et vise à donner une vision actualisée des croyances et des arguments qui font obstacle à la véritable diffusion de la théorie de Darwin.
\end{abstract}

Mots-clés: cognition, croyance, darwinisme, éducation, finalisme, raisonnement.

\begin{abstract}
Certain scientific theories are unable to impose themselves and become part of general common sense thinking and this has been the case for the Darwin's theory of evolution. This resistance varies in nature depending on the cultures involved (it is expressed differently in France and the United States, for example) but wherever it occurs it is underpinned by insidiously misleading lines of reasoning which are more or less implicit. This article is based on an in vitro experiment on 60 biology teachers subjects and aims to give an updated view of the beliefs and arguments which are obstacles to the veritable dissemination of Darwin's.
\end{abstract}

Keywords: beliefs, cognition, darwinism, education, finalism, reasoning. 


\section{INTRODUCTION La négligence de la taille de l'échantillon}

Une belle étude d’Andrew Shtulman et Joshua Valcarcel (2012) montre que certaines propositions scientifiques sont plus mal acceptées que d'autres. Leur expérience consistait à soumettre des individus à 200 questions couvrant dix domaines scientifiques (astronomie, génétique, thermodynamique, etc.), et à leur demander de répondre «vrai» ou «faux» à des énoncés du type: «la Lune produit de la lumière»; «I/I3 est plus grand qu’I/30»; «les atomes sont principalement composés de vide». Les sujets de l'expérience avaient tous rencontré, à un moment ou à un autre de leur parcours intellectuel, les réponses à ces questions et auraient dû savoir plutôt que croire. Les résultats de l'étude montrent que l'erreur n'est jamais si grande que lorsque il y a un conflit entre nos idées spontanées sur le monde et les propositions de la science. Ils montrent aussi que même lorsque les sujets ne se trompent pas, ils mettent, quoiqu'il en soit, plus de temps à répondre, ce qui est un indicateur de l'effort à produire pour résister à certaines intuitions.

Parmi toutes les propositions scientifiques difficilement acceptées par la logique ordinaire, la théorie de l'évolution a une belle place. D’une part, parce que c'est incontestablement l'une des théories les plus novatrices et importantes de l'histoire des idées. Et, d'autre part, parce que son caractère contreintuitif a été plusieurs fois remarqué (voir Guillo, 2009; Lecointre, 2009; Gould, 2006; Picq, 2006). À ce titre, de novembre 2005 à janvier 2006, nous avons mené $e^{1}$ une expérimentation dont le but était d'évaluer la façon dont des individus, titulaires au moins du baccalauréat, réagissaient face à une énigme de nature biologique qui pouvait être résolue en convoquant le programme darwinien. Les résultats obtenus montrent que les sujets de l'expérience ont des intuitions finalistes - qu'on pourrait dire crypto-lamarckiennes, parce que les motifs intellectuels recrutés ne se réfèrent pas clairement à une théorie explicite - et souvent guère compatibles avec la théorie de l'évolution ortho- 
doxe. Les conclusions que suggéraient ces résultats (Bronner, 2007), étaient que les oppositions théorie de l'évolution / finalisme pouvaient être idéologiques, comme aux États-Unis où elles convoquent des enjeux religieux (à travers la théorie du dessein intelligent ou du créationnisme), ou qu'elles pouvaient revêtir les atours (en France) d'un finalisme impensé qui se revendiquait du darwinisme, mais qu'elles devaient les unes et les autres être rapportées à un socle cognitif commun. Pour être plus précis, le caractère contre-intuitif de la théorie de l'évolution, quelles que soient les formes culturelles de son expression, est la conséquence d'une tentation inférentielle qui peut être dite négligence de la taille de l'échantillon (Bronner, 2006).

Il s'agit, en d'autres termes, de notre fréquente incapacité à tenir compte du nombre de phénomènes qui ont présidé à un fait que nous cherchons à apprécier. Cette erreur de raisonnement est d'autant plus attractive qu'elle concerne un phénomène à probabilité d'apparition faible, mais produit par un grand nombre d'occurrences. Nous avons, dès lors, l'impression qu'il est extraordinaire puisque nous ne pouvons, ou ne voulons pas, considérer la nature de la série de laquelle il est issu. C'est ainsi, par exemple, que certains veulent donner l'impression qu'ils ont un talent de voyant en produisant beaucoup de prédictions et en ne médiatisant que celles qui, par hasard, sont confirmées. De cet exercice, il peut ressortir, pour l'esprit non préparé, que la coïncidence entre la prédiction et l'événement, étant improbable en soit, ne peut être le fait que d'un pouvoir surnaturel. Il ne s'agit pourtant que d'une mise en scène visant à exhiber de maigres succès sans les comparer à la masse des insuccès. Si l'on permettait cette comparaison, l'illusion produite par la négligence de la taille de l'échantillon disparaîtrait. Ainsi, personne ne s'étonne de ce qu'il y ait des gagnants aux loteries, même lorsque les chances de gagner sont infimes. Pourquoi? Parce que chacun a clairement conscience que la taille de la population des joueurs et le nombre de grilles remplies sont immenses. Ici les probabilités sont faibles, mais elles sont intuitivement rapportées aux nombres d'occurrences impliquées. 
Cette illusion est donc une source très forte des croyances finalistes en biologie. En effet, on considère facilement que l'adaptation apparente de la vie à l'environnement montre que la nature ferait toujours «bien les choses». C'est donc bien le croisement de la fonctionnalité et du hasard qui paraît incommode au raisonnement finaliste qui peut, sous une forme stylisée, être rapporté à cette formule: la nature est si bien faite, cela ne peut pas être le fait du hasard. Il est vrai que la subtilité des entremêlements du monde vivant et les durées nécessaires à l'émergence de ces entremêlements sont tout simplement inimaginables à vue d'homme. La logique ordinaire focalise son attention sur les infimes probabilités de réussite du vivant, sans voir que ces probabilités sont à rapporter au nombre vertigineux d'expériences «ratées» de la nature. Dès lors, elle aura beaucoup de mal à percevoir la place qu'y occupe le hasard et proposera plus ou moins explicitement des hypothèses métaphysiques qui suggèrent qu'une entité (Dieu, une volonté supérieure, une force vitale, la Nature, etc.) a présidé au phénomène².

C'est cette erreur de raisonnement que nous avons pu voir à l'œuvre dans l'étude mentionnée plus haut. Dans cette étude, nous n’avions pris en compte que des individus ayant au minimum le baccalauréat pour s'assurer qu'ils avaient bien rencontré dans leur parcours intellectuel, même de façon superficielle, la théorie de l'évolution. Malgré cela, seuls I $8 \%$ arrivaient à concevoir et accepter une solution darwinienne dans un cadre expérimental dont je repréciserai les termes plus bas.

Il ne suffit donc pas d’avoir rencontré dans sa formation la théorie de l'évolution pour que l'obstacle intellectuel qu'elle représente disparaisse. Mais qu’en est-il lorsque cette formation est plus approfondie?

2 On retrouve ce type de raisonnement chez Michael J. Behe (1998), biochimiste américain, le biologiste néo-zélandais Michael Denton (1993) ou même chez le mathématicien William Dembski (2006), prosélyte du dessein intelligent, qui affirme, qu'en toute probabilité, la complexité de la constitution moléculaire des protéines, attendu qu'elles occupent un espace très faible des séquences polypeptidiques possibles, ne peut être que la conséquence d'une intention. 


\section{I.UN PROBLÈME D’ÉDUCATION OU DE COGNITION?}

L'erreur de raisonnement que j’ai nommée négligence de la taille de l'échantillon appartient à une catégorie de phénomènes mentaux que la psychologie de l'erreur nomme «biais cognitifs » ou «heuristiques» (Tversky, Kahneman, Slovic, 1984), «court-circuit mental» (Montgomery, 198I) ou encore «tunnel mental» (Piatelli Palmarini, 1995).

Dans les années 1970, Daniel Kahneman et Amos Tversky mirent sur pied, à l'université de Jérusalem, un ensemble d’expériences qui firent date. Très ingénieuses, ces expériences étaient amusantes et faciles à réaliser, les expérimentateurs n’ayant, la plupart du temps, qu’à présenter les énoncés apparemment anodins de quelques problèmes de logique ou de probabilité. Mais cela n'empêchait pas ces expériences de servir un projet scientifique ambitieux, puisque leurs inventeurs avaient pour but d'éclaircir le fonctionnement de la pensée humaine et, par là, de répondre à la question fondamentale : l'Homme est-il rationnel? En proposant une réponse à cette question, les deux psychologues visaient essentiellement une conception normative de la rationalité. C'est qu'une telle conception constituait alors l'orthodoxie ${ }^{3}$ en psychologie de la décision, elle pariait sur l'hypothèse selon laquelle les raisonnements individuels peuvent être modélisés efficacement en mobilisant les règles de la maximisation de l'utilité espérée. Par exemple, Kelley (1967) ou Peterson et Beach (1967) affirment que les raisonnements humains respectent les lois des statistiques et des probabilités ou encore Edwards (1968) considère que la logique naturelle suit grosso modo les prescriptions du théorème de Bayes. Or, les travaux de Tversky et Kahneman montreront indubitablement les limites de ce genre d'approche ${ }^{4}$.

3 À ce sujet, on peut lire la description décennie par décennie que proposent Marcus et Zajonc (1985) de l'évolution de l'idée de rationalité en psychologie.

4 Dès 1969, Tversky montre les faiblesses descriptives de l'axiome de transitivité, par exemple. Pour une synthèse plus large voir Tversky, Kahneman et Slovic (eds), 1984. 
Tversky et Kahneman ne sont certes pas les premiers à avoir travaillé sur ce type de problèmes ${ }^{5}$. Cependant, ils ont incontestablement joué un rôle essentiel dans la constitution d'une cartographie très développée de l'erreur humaine. Dans le fond, leur programme peut se résumer à une idée simple : face à des problèmes si complexes que nous n’avons ni les capacités ni le temps de les résoudre, nous recourons à des « recettes mentales » qui ont l'avantage de nous offrir des réponses rapides mais qui, dans certains cas, nous font commettre des erreurs.

Très tôt, certains se sont demandés si les résultats obtenus par les études sur les biais cognitifs ne relevaient pas tout simplement d'un problème d'éducation plutôt que de cognition. C'est notamment le cas du philosophe Jonathan Cohen (I98I) qui considère que l'on ne peut sérieusement s'attendre à ce que les individus ordinaires résolvent avec brio des problèmes dont ils ne pourraient trouver la solution que s'ils avaient des compétences en théorie des statistiques et probabilités. Il se trouve que dans un article intitulé «Belief in the Law of Small Numbers », et qui est antérieur au texte de Cohen, puisqu'il date de 197I, Tversky et Kahneman ont précisément montré que des spécialistes de statistiques pouvaient commettre des bévues cognitives équivalentes à celle de l'homme de tous les jours ${ }^{6}$. Le niveau d'étude ne constitue donc pas une protection définitive contre les erreurs cognitives, mais le problème posé par Cohen ne semble pas totalement épuisé. Nous savons peu de choses, en effet,

5 On pourrait mentionner les innombrables penseurs, Aristote, Cicéron, Bacon, Malebranche, Descartes, Condorcet et bien d'autres qui se sont donnés pour tâche de formaliser les façons de penser juste, en se méfiant du critère d'évidence et des pièges séduisants du sophisme. Dans ce tableau, il faudrait réserver une place particulière à John Stuart Mill (1988 [1843]). II faudrait sans doute ajouter les apports de Vilfredo Pareto dans son Traité de sociologie générale (1968 [1916]), la résolution du paradoxe de Saint Pétersbourg par Daniel Bernouilli en 1738, les remarques de Allais (1953) concernant le modèle du choix rationnel. Mais, à vrai dire, toutes ces contributions ne font que préfigurer les recherches menées par Amos Tversky et Daniel Kahneman à la fin $d u x x^{e}$ siècle, lesquels se reconnaissent des prédécesseurs: Paul Meehl(1954), et ses recherches sur la comparaison entre les prédictions cliniques et statistiques; Ward Edwards et son introduction, en psychologie, d'études sur la probabilité subjective dans le cadre du paradigme bayésien; Herbert Simon (1957) et le programme qu'il définit pour penser les stratégies du raisonnement; Jerome Bruner (1957) qui fut l'un des premiers à offrir une illustration empirique de ce programme; Fritz Heider (1944) et ses travaux pionniers sur la perception ordinaire de la causalité. 
sur la façon dont la formation intellectuelle augmente ou non notre résistance aux raisonnements captieux. Cette question paraît particulièrement intéressante à poser concernant la théorie de l'évolution car si les sujets de l'expérimentation que j’ai mentionnée plus haut avaient bien tous rencontré cette théorie dans leur formation, la plupart ne l’avaient pas beaucoup fréquentée intellectuellement. Dès lors, il m’a paru intéressant de faire passer «le test de l'éléphant» à des individus censés avoir une formation intellectuelle solide sur ce sujet et, qui plus est, chargés de l'enseigner à des élèves : les professeurs de science de la vie et de la terre (SVT) des collèges et lycées.

\section{RAPPEL DES FAITS CONCERNANT LA PREMIÈRE ENQUÊTE}

De novembre 2005 à janvier 2006, 60 entretiens furent menés auprès d'un échantillon caractérisé par son niveau d'étude (tous devaient être possesseur du baccalauréat) et par un critère de dispersion du point de vue de l'âge? Cette population vivait principalement en île de France $(\mathrm{N}=49)$, et tous en Métropole (Lorraine $\mathrm{N}=4$, Haute-Normandie $\mathrm{N}=4$, Midi-Pyrénées $\mathrm{N}=3$ ). Elle était composée de 33 femmes et 27 hommes, de cadres, professions intellectuelles et supérieures $(\mathrm{N}=\mathrm{I} 4)$, de professions intermédiaires $(\mathrm{N}=\mathrm{I} 7)$, d’employés $(\mathrm{N}=7)$, d'étudiants $(\mathrm{N}=\mathrm{II})$, de chômeurs $(\mathrm{N}=5)$, de retraités $(\mathrm{N}=4)$, d'un agriculteur exploitant et d'une femme au foyer.

Il s'agissait de confronter des individus volontaires, et dont l'anonymat était garanti, à une situation énigmatique tirée d’un fait scientifique réel ${ }^{8}$. La question posée était la suivante :

À l'état sauvage, certains éléphanteaux sont porteurs d'un gène qui prévient la formation des défenses. Les scientifiques ont constaté récemment que de plus en plus d'éléphanteaux naissaient porteurs de ce gène (ils n'auront donc pas de défenses devenus adultes). Comment expliquer cette situation?

7 Pour contrôler cette dispersion autour des valeurs centrales (l'âge moyen était de 37 ans), on rapportait l'intervalle interquartile à l'étendue. Le premier représentant plus de la moitié (59\%) de la seconde, on s'assurait ainsi d'éviter des phénomènes de concentration des âges.

8 En fait, ce mystère a été révélé et résolu par le professeur Zhang Li, zoologue à l'université de Pékin, qui a mené ses recherches depuis 1999 dans une réserve naturelle dans la région du sud-ouest de Xishuangbanna, où vivent les deux tiers des éléphants d'Asie chinois. 
La réponse à cette question était que les braconniers ne tuant pas les éléphants sans défenses (ceux-ci n’ont aucune valeur marchande pour eux), ces mutants sont plus nombreux dans la population et le gène qui prévient la formation des défenses se propage parmi les éléphants. Alors que ce gène se trouve habituellement chez 2 à $5 \%$ des éléphants d’Asie, on le trouve, à présent, chez 5 à $10 \%$ de la population des éléphants Chinois. Cette «énigme» trouve donc une solution acceptable en mobilisant certains processus sélectionnistes classiques de la théorie de l'évolution. Pourtant, assez peu d'individus sont capables de songer spontanément à ce type de solution, comme le montrent les résultats de l'enquête. La plupart des sujets de l'expérimentation optèrent pour une explication que l'on peut qualifier de finaliste. Ce finalisme pouvait prendre deux formes narratives. Soit l'interviewé affirmait que les défenses des éléphants ne leur servent plus, et que, devenues inutiles, elles tendent à disparaître. Soit il soulignait que les chasseurs tuent les éléphants pour leurs défenses d'ivoire, et que leur disparition constitue donc une adaptation de l'espèce «éléphant» à leur environnement : les éléphanteaux muteraient pour se protéger de la convoitise des chasseurs.

Le «finalisme» s'impose donc largement dans les discours alors que la solution darwinienne reste en retrait. En effet, les scénarios finalistes représentent 43,3\% des évocations globales et concernent $92 \%$ des interviewés (les interviewés avaient le droit de proposer autant d'hypothèses qu'ils le souhaitaient). Le scénario darwinien, lui, représente i2,6\% seulement des évocations globales et concerne $27 \%$ des interviewés. Parmi ces $27 \%$, $68 \%$ estiment que le scénario darwinien est le plus crédible. Par conséquent, seuls I8,3\% de l'effectif global parvient à concevoir et élire la solution darwinienne. 


\section{DU CÔTÉ DES SCIENCES DE LA VIE ET DE LA TERRE (SVT)}

De la même façon quaavec les titulaires du baccalauréat, cette expérimentation fut menée avec 60 enseignants de SVT du secondaire (collège et lycée). I7 entretiens ont été passés avec des enseignants de collège, 43 avec des enseignants de lycée. 3I avec des femmes, 29 avec des hommes. 28 ont été réalisés en Alsace et 3i en Île-de-France et I dans le Nord-Pas-de-Calais9.

Selon le même protocole, donc, les enseignants furent confrontés à l'énigme retranscrite par écrit (présentée plus haut). On leur demandait de le lire l'énoncé, puis, une fois qu'ils avaient déclaré l'avoir bien compris, l'entretien pouvait commencer. Comme dans la première expérimentation, la grille d'entretien avait été conçue pour inciter l'interviewé à donner toutes les réponses qui lui viendraient à l'esprit, attendu que ce sujet n'impliquait pas (en particulier en France), a priori, une charge idéologique ou émotionnelle forte, de nature à susciter des problèmes d'objectivation ou de régionalisation ${ }^{10}$. En revanche, contrairement à ce qui s'était produit avec la première expérimentation, les entretiens menés avec les enseignants de SVT donnèrent lieu souvent à des formes de revendication d’autorité. Avant d’accepter de tenter de résoudre l'énigme, les interviewés tenaient à signaler les approximations de l'énoncé et commencèrent dans près de $50 \%$ des cas (27 entretiens) par un cours sur la théorie de l'évolution.

Premièrement attention à votre expression; «gène» me dérange dans votre phrase : à remplacer par allèle [...] car si vous gardez gène cela veut dire qu'il existe deux populations d'éléphants qui diffèrent par le nombre de gènes (Extrait I : entretien avec un professeur de Lycée, homme, Île-de-France) ${ }^{11}$.

9 Je remercie ici la promotion 2006 de master I de sociologie de l'université Paris-Sorbonne et les étudiants de l'enquête inter-année de l'université de Strasbourg 2007 sans l'aide de laquelle cette recherche n'aurait pas été possible.

10 Voir Blanchet et Gotman, 1992.

II Cette remarque est tout à fait fondée. En fait le terme «gène» a été préféré lors de l'expérimentation avec les titulaires du baccalauréat parce que celui d'«allèle» est peu connu du grand public. Cette concession a entraîné, par volonté d'homogénéiser le protocole des deux expérimentations le fait de reprendre le terme de «gène». 
Sur un ton qui nous a paru parfois autoritaire, plus rarement méprisant, ils expliquaient à l'enquêteur dans quel cadre intellectuel devait être compris le problème qui leur était posé. C'est un point important car ce rapport de pouvoir structura en partie la nature des entretiens en deux moments. Le premier moment était «professoral» et le second (qui dans deux cas ne vint jamais, les entretiens ne purent être intégrés dans l'échantillon) «expérimental» (les enquêtés acceptaient de «jouer le jeu»). Ceci explique que, contrairement à ce que nous croyions initialement, les entretiens furent parfois difficiles à réaliser. Outre, les autorisations à obtenir de la part des proviseurs pour pouvoir pénétrer dans les établissements (l'intégralité des entretiens fut menée dans les établissements en face à face, sans observateur), plusieurs enseignants refusèrent de se plier à l'exercice pensant qu'on voulait exercer une sorte de contrôle sur eux et certains, lorsqu'ils acceptèrent, le firent avec beaucoup de méfiance car, s'ils ignoraient le sujet exact de l'expérimentation, ils devinaient qu'elle impliquait leur identité de professeur de SVT.

Cette méfiance est parfaitement compréhensible dans la mesure où il s'agissait, en effet, d'évaluer en partie leur compétence sur un sujet majeur de la connaissance biologique: la théorie de l'évolution. Ce rapport de pouvoir dans l'entretien a sans doute introduit un biais dans nos résultats. L'impact de ce biais est difficile à évaluer, mais on peut supposer qu'il a rendu les enseignants suspicieux et attentifs au fait de ne pas se tromper. Par conséquent, on peut faire l'hypothèse que, dans la quotidienneté de leur enseignement, les difficultés révélées par les résultats de notre recherche sont amplifiés plutôt qu'inhibés, ce qui les rend plus intéressants encore.

Je n'exposerai ici que deux des critères retenus pour analyser ces entretiens afin de permettre une comparaison claire avec les résultats obtenus avec les titulaires du baccalauréat.

Le critère d'évocation simple: il s'agit, comme son nom l'indique, de dénombrer les occurrences de l'apparition de tel ou tel type d'explication (sans tenir compte ni de l'ordre d’apparition ni de la récurrence de celle-ci dans le discours).

Le critère de crédibilité : à la fin de l'entretien, on demandait à l'interviewé celui qui lui paraissait le plus crédible d’entre les scénarios qu’il avait évoqués. On demandait par exemple: «Si vous aviez à parier sur l'une des solutions de l'énigme que vous avez proposées, laquelle ferait l'objet de votre mise?». On 
laissait la possibilité aux interviewés qui le désiraient de parier sur deux hypothèses (dans ce cas, on attribuait à chacune d'elle un demi-point).

Alors que les titulaires du baccalauréat évoquent en moyenne 2,12 scénarios, les enseignants de SVT en évoquent 2,I8. Cette similarité peut éventuellement constituer une surprise car on aurait pu sattendre à ce que les enseignants ne mobilisent qu’un scénario, celui d’un processus sélectionniste et s'en tiennent là. Pour le dire autrement, la compétence aurait pu inhiber l'imagination, mais ce ne fut pas le cas.

Dans leurs discours, les scénarios finalistes représentent 32,8\% des évocations globales et concernent $72 \%$ des interviewés. Le scénario darwinien, lui, représente $29,7 \%$ des évocations globales et concerne $65 \%$ des interviewés. Parmi ces $65 \%, 77 \%$ estiment que le scénario darwinien est le plus crédible. Par conséquent, $50 \%$ de l'effectif global parvient à concevoir et élire la solution darwinienne.

Si l'on prend en compte l'ensemble des scénarios évoqués et que l'on compare les résultats obtenus par les titulaires du baccalauréat et les enseignants de SVT au test de l'éléphant, on obtient:

Figure I : le critère d'évocation simple enseignants SVT / baccalauréat

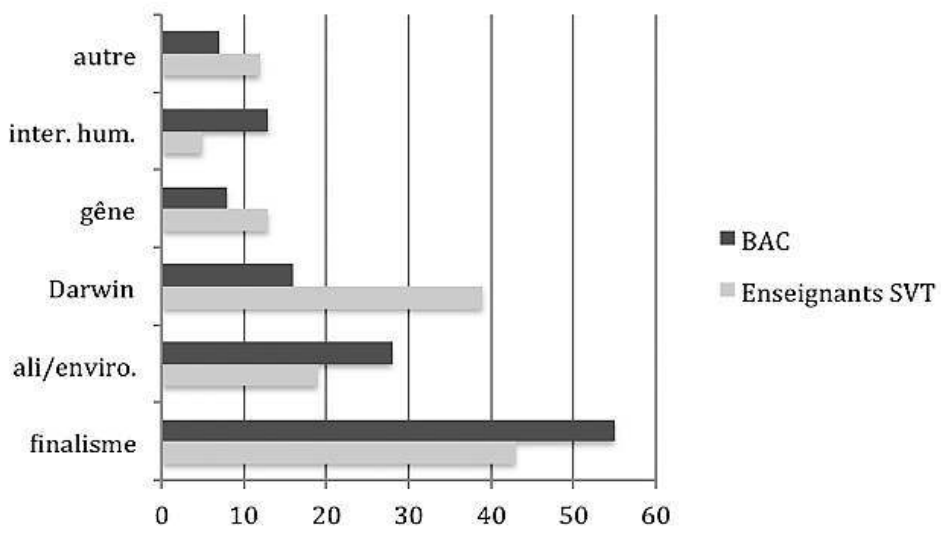


La typologie utilisée pour classer les discours se fonde sur le mode d'explication choisi par les interviewés pour résoudre l'énigme proposée. J’ai précisé plus haut ce qu'il fallait entendre par «explication finaliste » et ce qui était considéré comme une «solution darwinienne $»^{12}$. Concernant le type «alimentation/environnement», il s'agissait, pour les interviewés, de résoudre l'énigme en invoquant une modification de l'environnement et/ou de l'alimentation de la population des éléphants. Par exemple :

Une mutation génétique, ça peut venir d'un élément extérieur, style pollution. Peut-être que le milieu ne le permet plus de fabriquer leurs défenses. Le gène ne peut pas fonctionner sans matière première. C'est-à-dire que les éléments ne trouvent plus la nourriture qui leur permet de fabriquer leurs défenses à cause, je sais pas, de la disparition de certains végétaux dont ils se nourrissaient, par exemple, la pollution (Extrait 2, titulaire du baccalauréat, homme).

Concernant le type «gène dominant», comme son nom l'indique, il propose de résoudre l'énigme en considérant que le gène qui prévient la formation des défenses est «dominant», donc il tend à se répandre dans la population des éléphants. Certains sujets proposent alors une solution hybridée en soulignant que ce gène est apparu récemment à cause d'une modification dans l'environnement ou l'alimentation par exemple.

Sous l'intitulé «intervention humaine» ont été placés les modes d'explications qui considéraient qu'une intervention humaine est à l'origine de la situation énigmatique. Par exemple :

Ou alors, c'est peut-être les U.S.A. qui leur ont inoculé un truc, pour contrôler la contrebande. Un truc pour que les éléphants n’aient plus de défenses, pour qu'ils se fassent plus chasser (Extrait 3 : titulaire du baccalauréat, homme).

Enfin, on a placé dans «autres» tous les récits ne correspondant pas aux types les plus fréquemment rencontrés. La plupart du temps, il s’agit de propositions uniques par leur type. Par exemple:

12 La réponse découverte par Zhang Li ne fut pas la seule à être considérée comme darwinienne évidemment. Par exemple, l'idée que les éléphants sans défense pouvaient paraître plus «séduisants » aux partenaires de l'autre sexe et donc s'accoupler plus facilement, pour farfelue qu'elle paraisse, respecte le principe sélectionniste. 
Comme les défenses sont un signe d’agressivité, de self-défense, d’agressivité, d’attaque etc. et comme les éléphants sont des animaux très sociaux, ils se sont rendus compte, en fait, qu'il y avait pas mal de morts, même entre eux, dues à des accidents ou dues à des attaques impromptues entre les éléphants et bien... effectivement, si eux retirent leur défense d'éléphants, les hommes pourraient pas aussi arrêter de se battre entre eux? Faire qu'il n'y ait plus d'armes sur la Terre pour, comme les éléphants, les hommes se tuent entre eux. Donc c'est un signe de paix, c'est la Nature qui montre exemple à l'Homme sur comment ne pas se battre (Extrait 4 : titulaire du baccalauréat, femme).

\section{CONCURRENCE INTRA-INDIVIDUELLE}

La fréquentation d'une théorie contre-intuitive, en l'occurrence la théorie de l'évolution, permet donc de limiter l'influence d'un raisonnement captieux (ici le finalisme adossé à la négligence de la taille de l'échantillon), mais ne le fait pas disparaître, loin s'en faut. Ces résultats sont conformes aux études disponibles sur ce sujet (Ericsson et al., 2006; Bronner, 2008). Ils sont incommodes car on peut tout aussi bien y voir, selon la métaphore du verre à moitié vide ou à moitié plein, les signes encourageants de la possibilité de faire reculer, par l'éducation, les intuitions trompeuses qui se rendent disponibles à notre esprit dans certaines circonstances ou, tout au contraire, les indices inquiétants de l'impossibilité de réduire à un niveau acceptable leur pouvoir de nuisance. En effet, non seulement, seul un enseignant sur deux parvient à proposer et valider une solution darwinienne du problème, mais $72 \%$ d'entre eux envisagent comme hypothèse possible une solution finaliste. On aurait pu s'attendre à ce qu'une formation académique disciplinaire désactive l'évocation même de solutions lamarckiennes. Ce qui ne fut pas le cas. On ne peut pas dire non plus que ces résultats s'expliqueraient par l'existence de deux sous-populations distinctes : les compétents qui convoqueraient une solution sélectionniste et les autres qui s'abandonneraient à une explication finaliste. En fait, seuls I $8 \%$ des professeurs de SVT interrogés ont proposé une réponse fermement darwinienne sans se laisser tenter par des explications moins satisfaisantes. Ces entretiens furent typiquement les plus courts, les sujets manifestaient une grande confiance dans leur réponse unique et ne souhaitaient pas proposer 
une autre solution à l'énigme. Une autre partie, I0 \%, optait pour une solution darwinienne mais après quelques tâtonnements, une autre encore, impliquant $37 \%$ des interviewés, mettait clairement en concurrence des options sélectionnistes et finalistes. Une dernière partie enfin, 35\% des interviewés, n’envisageaient pas la solution darwinienne, mais pouvait tout de même ressentir une forme d'inconfort quant à l'option finaliste. L'extrait qui suit montre typiquement comment cet inconfort peut s'exprimer :

Enseignant I SVT : Bah... je dirais que... que... ça rejoint la théorie de l'évolution, et que donc ça doit être... soit une mutation qui... qui se, qui est dominante, et donc qui se répand beaucoup, ou... ou alors, enfin... [rires]... si jamais les éléphants se servent de moins en moins de leurs défenses... ben oui ça peut conduire justement à des mutations et... au fait que... peut-être que les éléphants... ils... ouais je sais pas... C'est comme les humains ils ont de moins en moins de dents. On voit de plus en plus d'enfants qui naissent justement avec des dents en moins. Donc... euh... ouais je pense que c'est l'évolution. [...] Euh... bah... peut-être quavant ça leur servait à se battre, et maintenant ils deviennent gentils ? (Rires)... je sais pas! Il doit y avoir quelque chose qui a changé dans leur comportement ou dans leur environnement pour que... ben oui pour qu'ils n'aient plus besoin de leurs défenses, et du coup il se développe un gène qui prévient l'apparition des défenses parce que si elles ne leurs servent à rien... ben ils ont pas besoin d'en avoir! Et du coup... elles se développent de moins en moins chez les éléphanteaux... Parce que c'est le sens de l'évolution, au fil du temps... il ne reste chez les êtres vivants que ce qui leur est utile ou ce qui les rends plus performants contre les prédateurs ou pour survivre dans leurs milieux de vie [...].

Interviewer : Vous voyez d’autres explications?

Enseignant SVT I : Euhm... ah, le braconnage !

Interviewer : C'est-à-dire ?

Enseignant SVT I : Ben euh... il y avait des braconniers qui tuaient les éléphants. Pour euh... pour leurs défenses, pour l'ivoire... et euh... peut-être qu'on peut trouver euh... que... on peut se dire que pour prévenir la mort des éléphants ils se sont dit «hop on va faire un gène!» [rires], j'en sais rien! Euh... Une sorte de sélection naturelle... je sais pas trop comment expliquer ça mais... ouais, peutêtre que ça sert à prévenir l'extinction des éléphants due au braconnage... J'en sais rien parce que c'est bizarre, les éléphants ne vont pas se dire «tiens, il y a 
les braconniers qui nous tuent » alors... Je m’emmêle les pinceaux... Les éléphanteaux ne voient pas les braconniers qui vont les tuer... Euh... Peut-être... inconsciemment? Je sais pas... c'est bizarre... Ce qui est sûr c'est qu'il ne suffit pas de penser quelque chose pour que ça se répercute sur les gènes et l'ADN, heureusement en fait parce que les mutations seraient trop rapides et partiraient dans tous les sens! Mais oui donc une mutation qui ferait... pour prévenir la mortalité due au braconnage. C'est un exemple mais... enfin c'est une explication qui me vient à l'esprit! Mais c'est vrai que... alors donc il y a une forte mortalité due au braconnage... alors les éléphanteaux ne font plus de défenses... ben oui c'est logique, c'est pour survivre, ils s'adaptent au milieu parce qu'ils savent qu'avec des défenses ils ont plus de chances de mourir alors... les défenses ne se développent plus dans la population (Extrait 5, enseignant de SVT, collège, femme).

Cette enseignante a clairement conscience que la solution qu'elle choisit n'est pas optimale du point de vue cognitif, ses hésitations, ses rires, ses déclarations même en attestent, mais elle paraît s'en satisfaire de guerre lasse. Dans certains autres entretiens, l'enseignant est souvent à deux doigts de défendre une option sélectionniste, mais la tentation finaliste finit par l'emporter. Ainsi, cet autre extrait est non moins intéressant parce que l'interviewé inaugure l'entretien par les précisions qui lui paraissent devoir être faites et une introduction très brève à la théorie de l'évolution :

Enseignant SVT 2 : Bon, euh... je vais recommencer en essayant d'être un peu plus précis. En gros, le fait est que... si jamais... Les gènes subissent des mutations qui provoquent une nouvelle allèle... et cette allèle soit elle disparaît, soit elle se propage dans toute la population. Si il disparaît... c'est alors qu'il, ben c'est qu'il est désavantageux pour l'espèce, c'est-à-dire, ben en gros qu'il n’a plus d'utilité... enfin qu'il sert à rien quoi... Et au contraire... s'il se développe c'est qu'il est avantageux pour l'espèce, donc là il est utile à quelque chose, il est utile pour la survie des éléphants, pour le développement de l'espèce. Et ça veut dire que ce gène-là ben justement est avantageux (Extrait 6, enseignant de SVT 2, Lycée, homme).

Jusque-là, malgré l'ambiguïté du vocabulaire utilisé (notamment les termes «utile» «utilité ») il est encore possible d'énoncer une hypothèse sélectionniste. Le début de cet entretien est d’ailleurs représentatif du scénario type que nous avons rencontré : il sagit pour les interviewés de montrer qu’ils connaissent la théorie de l'évolution (ce dont ils s'acquittent convenablement le plus souvent), mais dès 
lors qu'il s'agit d'appliquer cette théorie au cas concret qui est proposé, une partie d'entre eux s'abandonnent au finalisme avec la complicité de termes pièges comme ceux de «fonction», d'«utilité», etc. La suite de cet entretien le montre :

Enseignant SVT 2: C'est la sélection naturelle des gènes, si certains gènes, ou les allèles qui le composent ne servent pas à l'espèce ou pire sont mauvais pour elle... enfin lui apportent des désavantages comme euh... courir pas assez vite, avoir des muscles pas assez puissants... ce genre de choses quoi... ben du coup... ces gènes ne vont pas se propager dans la population parce que c'est pas ce qu'il y a de mieux pour elle, et ce qui se propage... enfin les gènes qui se développent c'est ceux qui ont été choisis par la sélection naturelle... parce que... euh... ben c'est les meilleurs par rapport aux autres gènes quoi. C'est la sélection naturelle qui détermine les gènes parce qu'elle garde ceux qui sont avantageux et pas ceux qui sont désavantageux, comme ça dans la population, à terme... enfin si tout se passe comme prévu... il ne restera que les gènes les plus avantageux. Et là peut-être que les défenses ne sont pas avantageuses pour l'espèce alors ce gène se développe pour que les éléphanteaux n'en aient plus... Enfin je vois ça comme ça moi (Extrait 6, enseignant de SVT 2, Lycée, homme).

Après plusieurs tentatives d'explorer d'autres hypothèses sans parvenir à proposer un mécanisme darwinien, l'enseignant revient sur son explication première, persuadé qu'il s'agit là d’une interprétation orthodoxe.

Enseignant SVT 2: Parce que pour moi la sélection naturelle c'est la réponse à ce genre de questions, je pense que c'est ce qui est le plus clair... et logique et... ouais je vois rien d'autre. Enfin si après y à des explications comme le dessein intelligent ou pire le créationnisme mais ça pour moi c'est pas scientifique... parce que dire que c'est Dieu qui a fait que les choses sont comme ça c'est trop facile, pas besoin de se casser la tête à trouver une explication hein! [rires] Ouais... je pense vraiment que c'est pas scientifique (Extrait 7, enseignant de SVT 2, Lycée, homme).

La formation intellectuelle de ces enseignants de SVT ne fait donc pas disparaître la concurrence intra-individuelle qui résulte de la coexistence dans notre esprit de l'hypothèse sélectionniste et de l'hypothèse finaliste. En revanche, elle permet de donner statistiquement une accessibilité plus fluide au scénario darwinien. 
Cette concurrence met en scène une tension classique entre une solution optimale et une solution satisfaisante. Comme beaucoup d'auteurs l'ont fait remarquer, on pense tout particulièrement à la notion de rationalité procédurale chez Herbert Simon (1959, I963) ${ }^{13}$, les individus choisissent souvent plutôt que l'inférence optimale, une inférence satisfaisante. Dans le fond, les acteurs sociaux acceptent certaines explications objectivement douteuses parce qu'elles paraissent pertinentes dans le sens que Sperber et Wilson (1989) ont donné à ce terme. En situation de concurrence, expliquent-ils, on optera pour la proposition qui produit le plus d'effet cognitif possible pour le moindre effort mental. La solution objectivement bonne, lorsqu'elle existe, est souvent la plus satisfaisante (en l'occurrence, lorsque les interviewés parviennent à concevoir une solution darwinienne, c'est bien elle très majoritairement qu'ils considèrent comme crédible), mais les individus non pas toujours assez d'imagination et de motivation pour cela et s'abandonne fréquemment à ce que Fiske et Taylor (1984) nomment notre « avarice cognitive». Cette «avarice cognitive» nous détourne de certaines procédures de contrôle mental. En d'autres termes, parce que la solution finaliste est satisfaisante et intuitive, elle exerce une grande attraction sur les avares cognitifs que nous sommes. Mais une formation disciplinaire adéquate permet de réduire le coût mental de la solution darwinienne qui devient plus concurrentielle alors.

\section{UN COÛT MENTAL}

C'est que ces contrôles mentaux sont coûteux, et d'une façon qui n'a rien de métaphorique. On l'a vu dans l'introduction de cet article, avec l'étude d'Andrew Shtulman et Joshua Valcarcel (20I2), même lorsque les sujets parviennent à trouver la bonne réponse, ils mettent plus de temps pour les propositions contre-intuitives (et on constate là aussi que l’acquisition précoce de la culture scientifique n'empêche pas totalement les intuitions trompeuses, mais les inhibe notablement, les auteurs de cette étude le soulignent). Le temps est ici un indicateur possible de ce que nous coûte, en termes d'énergie mentale, la lutte contre les limites cognitives de la rationalité. Mais l'imagerie 
cérébrale permet d’aller plus loin. Boehler et son équipe (201 I), par exemple, ont pu montrer que l'anticipation d'une tâche (il s'agissait d'un exercice de discrimination visuelle) nécessitant de grands efforts mobilisait plus de régions cérébrales qu'une tâche de même type nécessitant moins d'efforts. D’ailleurs, d'autres études (McGuire, Botvinick, 20ı) ont permis d'identifier une région cérébrale dévolue à l'évaluation de la valeur d'ensemble d'une action accomplie, ou d'un investissement mental. L'effort paraît y être considéré comme un coût dans ce qu'on pourrait appeler un calcul neural. Ce coût est particulièrement important lorsqu'il s'agit de passer d'une tâche mentale à une autre, comme l'a montré Stephen Monsell (2003), ce que réclame exactement le fait de changer de cadre cognitif pour résoudre une énigme. Cet effort intellectuel, se traduit notamment par une consommation de glucose. Notre système nerveux est, en effet, plus consommateur de cet aldohexose que tous les autres organes du corps humain et il se trouve qu'une activité mentale ardue est coûteuse en glucose (voir Kahneman, 20I2, p. 56).

À partir du milieu des années 1990, plusieurs études (Smith, McEvoy, Gevins, 1999; Raichle, et al., 1994; Berthoz, 2003) ont montré que l’apprentissage limitait les coûts énergétiques d’une activité mentale. En d’autres termes, plus la compétence d’un individu se développe, moins les régions cérébrales impliquées sont nombreuses. Typiquement, un apprentissage qui est d'abord traité comme un objet au niveau du cortex préfrontal s’accompagne d'un désengagement progressif des structures corticales à mesure que ces objets deviennent des routines mentales.

Deux hypothèses spéculatives peuvent être formulées pour rendre compte des résultats obtenus par les enseignants de SVT au test de l'éléphant.

1. La fréquentation d'une théorie permet de rendre plus spontané (donc moins coûteux du point de vue de l'énergie mentale) le recours, non peutêtre à la théorie en elle-même, mais à son socle cognitif (ici les mécanismes sélectionnistes). Lorsque cette théorie n’est pas contre-intuitive (ou plus exactement lorsqu'elle náa pas à subir la concurrence de propositions alternatives plus intuitives), elle pourra même s'exprimer sous des formes virtuoses chez l'individu. Lorsqu'elle est contre-intuitive, son apprentissage permettra de la rendre plus concurrentielle par rapport à d'autres propositions bénéficiant d'une grande force d'évocation spontanée. 
2. La fréquentation d'une théorie ne permet pas de la rendre plus spontanée, mais aiguise un réflexe de méfiance face à une alternative satisfaisante mais non optimale. L'individu a appris à reconnaître une situation «piégée ». Le coût nécessaire à la convocation de la théorie contre-intuitive reste important, mais l'individu est près à l'assumer (il s'agit d'une façon métaphorique de décrire le processus bien entendu car cette décision ne relève du traitement pleinement conscient que dans des cas limites). Comme le précise Kahneman (2012, p. 55) : «Lors de plusieurs expériences les gens parvenaient à résister aux effets de l'épuisement de l'ego, quand on leur donnait une bonne raison de le faire».

\section{CONCLUSION \\ Des enjeux pédagogiques}

Ces résultats expérimentaux intéresseront, je l'espère, la sociologie de la connaissance (prise en son acception large), dans la mesure où ils éclairent, par un exemple, la façon dont la formation intellectuelle peut concurrencer ou non le caractère captieux de certaines tentations inférentielles. Mais ils me paraissent pointer aussi des enjeux pédagogiques.

D'une part, parce que la théorie de l'évolution constitue le socle de la représentation orthodoxe du monde du vivant. Par conséquent, la bonne connaissance des obstacles intellectuels qui s'opposent à sa réception spontanée et à son enseignement constitue un enjeu suffisant pour que l'on s'y penche. Cet enjeu est redoublé par le fait qu'une nouvelle contestation de cette théorie paraît se faire jour, en France. En effet, certains élèves au nom de considérations religieuses (notamment chez les jeunes musulmans ${ }^{14}$ ) remettent en question l'enseignement de cette théorie. Ces cas de figure, même s'ils sont rares, imposent au pédagogue la parfaite maîtrise de l'illusion intellectuelle que représente le finalisme.

14 Voir par exemple l'interview de Jean-Baptiste de Panafieu sur ce point, même si, à ma connaissance, il n'existe pas d'étude méthodique sur ce sujet: <http://sciencesetavenir.nouvelobs.com/archeo-paleo/20090122.OBS0939//-evolution-contestee-dans-les-ecoles.html>. 
D’autre part, parce que la théorie de l'évolution n'est pas la seule à être contre-intuitive dans les divers enseignements que les élèves auront à appréhender. Il se trouve que nous commençons à connaître assez bien la cartographie de nos erreurs systématiques (ce que les psychologues nomment les «biais cognitifs »). Elle est sans doute encore incomplète, mais elle permet d'engager une réflexion inédite sur les modes d’apprentissage. Ces «illusions mentales » peuvent surgir dans tous les domaines de la connaissance : physique, biologie, mathématique, sciences économiques et sociales, histoire, philosophie, etc. En réalité, les programmes pédagogiques en sont constellés sans que les pédagogues les aient systématiquement repérées ou prises au sérieux. Du moins les manuels tels qu'ils sont conçus n'indiquent pas une volonté particulière de mettre à jour ces obstacles intellectuels.

L’identification systématique des entraves cognitives à la réception spontanée de certaines théories et la compréhension des processus contre-intuitifs qu'ils impliquent permettraient d'engager l'apprenant dans une auto-analyse de ses difficultés à comprendre. Creuser le sillon de la pensée méthodique pour que chacun soit en mesure de se méfier de ses propres intuitions, identifier les situations où il est nécessaire de suspendre son jugement, d'investir de l'énergie et du temps plutôt que d'endosser une solution qui paraît acceptable, en un mot dompter l'avare cognitif qu'il y a en nous tous, tel pourrait être le programme de cette réflexion pédagogique. L’enseignement de toute chose doit tenir compte, pour être efficace, des caractéristiques du récepteur du message. Or, il est des caractéristiques si partagées et invariantes que l’apprentissage ne saurait en faire l'impasse. 


\section{BIBLIOGRAPHIE}

ALLAIS M., 1953, «Le comportement de l'homme rationnel devant le risque: critique des postulats et axiomes de l'école américaine», Econometrica, 21-4, p. 503-546.

BEHE M. J., 1998, Darwin's Black Box: The Biochemical Challenge to Evolution, New York, Free Press.

BERNOULLI D., 1954 (1738), «Exposition of a New Theory on the Measurement of Risk», Econometrica, XXII, p. 23-36.

BERTHOZ A., 2003, La Décision, Paris, Odile Jacob.

BLANCHET A., GOTMAN A., 1992, L'Entretien, Paris, Nathan.

BOEHLER C.N., et al., 20 II, «Task-Load-Dependant Activation of Dopaminergic Midbrain Areas in the Absence of Reward », Journal of Neuroscience, 31, p. 4955-4961.

BRONNER G., 2006, Vie et mort des croyances collectives, Paris, Hermann.

-, 2007, «La résistance au darwinisme: croyances et raisonnements », La Revue Française de Sociologie, 48-3, p. 587-607.

-, 2008, «Les normes du raisonnement: entre inné et acquis», Philosophia Scientae, 9 , p. $193-213$.

BRUNER J., 1957, «Going Beyond the Information Given», in Contemporary approaches to cognition, H.E. Gruber et al. (eds), Cambridge, Harvard University Press, p. $41-69$.

COHEN J., 1981, «Can Human Irrationality Be Experimentally Demonstrated?», Behavorial and Brain Sciences, 4, p. 317-370.

DEMBSKI W.A., 2006, The Design Inference: Eliminating Chance Through Small Probabilities, Cambridge, Cambridge University Press.

DENTON M., 1993, Évolution: une théorie en crise, Paris, Flammarion.

EDDY D., 1984, «Probabilistic Reasoning in Clinical Medecine: Problems and Opportunities», in Judgment under Uncertainty: Heuristics and Biaises, A. Tversky, D. Kahneman and P. Slovic (eds), Cambridge, Cambridge University Press, p. $249-267$. 
EDWARDS W., 1968, «Conservartism in Human Information Processing», in Formal Representation in Human Judgement, B. Kleinmutz (ed.), New York, Wiley, p. 17-52.

ERICSSON K. ANDERS et al., 2006, The Cambridge Handbook of Expertise and Expert Performance, New York, Cambridge University Press.

FISKE S.T., TAYLOR S.E., 1984, Social cognition, New York, Random House.

GOULD S. J., 2005, La Structure de la théorie de l'évolution, Paris, Gallimard.

GUILLO D., 2009, Ni Dieu, ni Darwin. Les Français et la théorie de l'évolution, Paris, Ellipses.

HEIDER F., 1944, «Social Perception and Phenomenal Causality», Psychological Review, 5I, p.358-373.

KAHNEMAN D., 2012, Système I, Système 2, Paris, Flammarion.

KELLEY H.H., 1967, «Attribution theory in social psychology», in Nebraska Symposium on Motivation, D. Levine ed., Nebraska, University of Nebraska Press, p. 192-238.

LECOINTRE G. (éd.), 2009, Guide critique de l'évolution, Paris, Belin.

MARCUS H., ZAJONC R.B., 1985, «Cognitive perspective in social psychology», in Handbook of social psychology, vol. I, G. Lindzey et E. Aronson (eds), New York, Random House, p. 137-230.

MCGUIRE J.T., BOTVINICK M.M., 20I0, «Prefrontal Cortex, Cognitive Control, and the Registration of Decision Costs», PNAS, 107, p. 7922-7926.

MEEHL P., 1954, Clinical versus Statistical Prediction: A Theorical Analysis and a Review of the Evidence, Minneapolis, University of Minnesota Press.

MILL J. S., 1988 (I843), Système de logique, Bruxelles, Mardaga.

MITTROF H., 1974, The Subjective Side of Science, Amsterdam, Elsevier.

MONSELL S., 2003 «Task Switching», Trends in Cognitive Sciences, 7, p. I34-140.

MONTGOMERY H., 1983, «Decision Rules and the Search for a Dominance Structure: Toward a Process Model of Decision Making» in Analysing and aiding decision processes, P. Humphreys, O. Svenson and A. Vári (eds), Budapest, Publishing House of Hungarian Academy of Sciences, p. 343-369. 
PARETO V., 1968 (1916), Traité de sociologie générale, Fuvres complètes, t.XII, Genève, Droz.

PETERSON C.R., BEACH L.R., 1967, «Man as an Intuitive Statistician», Psychological Bulletin, 68, p. 29-46.

PIATELLI PALMARINI M., 1995, La Réforme du jugement ou comment ne plus se tromper, Paris, Odile Jacob.

PICQ P., 2009, Darwin et l'évolution expliqués à nos petits enfants, Paris, Seuil.

RAICHLE M. et al., 1994, «Practice-Related Changes in Human Brain Functional Anatomy during Nonmotor Learning», Cerebral Cortex, 4, p. 8-26.

SIMON H., 1957, Models of Man: Social and Rational, New York, Wiley.

-, 1959, «Theories and Decision-Making in Economics and Behavioral Science», American Economic Review, 49-3, p. 253-283.

-, 1963, «Economics and Psychology» in Psychology: A Study of Science, t.IV, S. Koch (ed.), New York, McGraw Hill, p. 685-723.

SHTULMAN A., VALCARCEL J., 2012, «Scientific Knowledge Suppresses but Does Not Supplant Earlier Intuitions» Cognition, 124-2, p. 209-2I5.

SMITH M.E., MCEVOY L.K., GEVINS A., 1999, «Neurophysiological Indices of Strategy Development and Skill Acquisition», Cognitive Brain Research, 7, p. 389-404.

SPERBER D., WILSON D. 1989, La Pertinence - Communication et Cognition, Paris, Éditions de Minuit.

TVERSKY A., 1969, «Intransitivity of Preferences», Psychological Review, 76, p. 31-48. -, KAHNEMAN D., 197I, «Belief in the Law of Small Numbers», Psychological Bulletin, 2, p. 105-110.

TVERSKY A., KAHNEMAN D., SLOVIC P. (eds), 1984, Judgment under Uncertainty: Heuristics and Biaises, Cambridge, Cambridge University Press. 
